\section{Paths of yeast assistance}

\section{By Michael J. Haas, Senior Writer}

Many natural products have pharmacological activities of potential therapeutic interest, but research has been hampered by problems producing enough pure material for research purposes. In Nature Chemical Biology, researchers at the California Institute of Technology now have described an alkaloid production platform that uses engineered yeast for the customized biosynthesis of natural products. ${ }^{1}$

In proof-of-concept studies with benzylisoquinolines, a large family of plant alkaloids, the platform achieved product yields in the range of tens of $\mathrm{mg} / \mathrm{L}$, which are several times higher than those achieved with conventional approaches that rely on plant cultivation or laboratory synthesis.

Researchers expect the platform could support the development of natural products by generating them on a scale suitable for preclinical and clinical trials. Companies in the botanicals and natural products space contacted by $S c i B X$ agreed with this assertion, adding that the platform has much potential as a drug discovery engine for natural products and their derivatives.

\section{Exploring space with yeast}

Christina Smolke, assistant professor of chemical engineering at Caltech, and Kristy Hawkins, a graduate student in Smolke's lab, developed the yeast-based platform. The limitations of existing plantand synthetic-based approaches motivated Smolke to reconstruct the alkaloid-producing metabolic pathways in plants in a single microorganism - a strain of the yeast Saccharomyces cerevisiae.

"Only a limited number of possible alkaloid structures can be studied in plants," Smolke told SciBX. "But it's predicted that there are about 2,500 pharmacologically interesting structures on the plant metabolic pathways that produce benzylisoquinoline alkaloids."

Smolke said one advantage of producing the compounds in yeast instead of extracting them from plants is that the downstream process is simpler. "You get more reliable and more consistent product output, and easier recovery because the yeast excrete the compounds right into the growth medium," she said.

In addition, producing the compounds in a single microorganism offers advantages over coculture systems, according to Smolke. In such systems, different portions of an alkaloid biosynthetic pathway are reconstructed in two or more separate microorganisms.

A recent report by a research team at Ishikawa Prefectural University, for example, describes the reconstruction of the first portion of the benzylisoquinoline alkaloid pathway in Escherichia coli and the final steps toward the biosynthesis of complex alkaloids in yeast. ${ }^{2}$

Smolke said such coculture systems take a hit in yield because one microorganism has to transfer its metabolic product to the other microorganism to complete the process. That hand-off, she said, can significantly limit overall yields.

The yields in the Ishikawa study were below $10 \mathrm{mg} / \mathrm{L}$ - not substantially different from those achieved by synthesis or plant extraction.

Indeed, Smolke said "alkaloids don't cross cell membranes well. They are not natural metabolites in the microorganisms, so the microorganisms don't have transporters for them." By contrast, she said the single-culture yeast system that she and Hawkins developed avoids this issue and achieves 10-100 times better yields.

Smolke and Hawkins first engineered S. cerevisiae to express three plant enzymes that produced alkaloids in well-studied plants, such as the opium poppy Papaver somniferum. The authors then supplemented the yeast cultures with norlaudanosoline-a commercially available alkaloid precursor-which the yeast metabolized to reticuline, a key alkaloid intermediate and a precursor to benzylisoquinolines.

Before engineering the yeast to convert reticuline into other alkaloids, the researchers sought to maximize reticuline yield with minimum interference in the natural life cycle of the yeast. They did so by identifying the optimum level for each of the three enzymes in the system.

"Our lab previously described this strategy but did not describe its specific application to tuning enzyme levels," Smolke said. ${ }^{3}$

The optimization of enzyme expression is important, she said, because expression of heterologous enzymes requires yeast to divert resources away from its own growth and reproductive processes. Moreover, higher expression of enzyme will only increase product yield up to a point-above a certain level, there is no appreciable increase in yield.

After optimizing the platform's yield, the two researchers engineered the yeast to convert reticuline into other alkaloids. They achieved this by expressing the three enzymes required to produce reticuline and up to four other enzymes derived from alkaloid-producing plants that were known to convert reticuline into alkaloids along two specific branches of the benzylisoquinoline alkaloid pathway. Depending on which combination of enzymes the researchers selected, the yeast produced different alkaloids along those metabolic pathways.

Lastly, Smolke and Hawkins showed their platform could produce alkaloids using enzymes from sources other than alkaloid-producing plants. They were interested in human cytochrome P450, family 2, subfamily D, polypeptide 6 (CYP2D6) because a 2005 study suggested CYP2D6 plays a role in producing small amounts of morphine in human cells. ${ }^{4}$

Indeed, Smolke and Hawkins found that yeast expressing CYP2D6 and the three plant enzymes required to produce reticuline could convert reticuline to salutaridine, an early intermediate along the branch of the benzylisoquinoline pathway that leads to morphine. 
Smolke said the result obtained with CYP2D6 "highlights the fact that you can mix and match enzymes from different sources-including those not native to the pathway-and still produce molecules of use and interest."

She also said it demonstrates that the platform is a tool not only for production, but also for characterizing metabolic pathways. "For instance, one could do functional genomics screens of plant enzyme libraries to identify which ones have activity on a given pathway," she said.

The authors wrote that their results show the engineered yeast platform can readily produce pharmacologically interesting molecules that are difficult, if not impossible, to produce in other ways.

Smolke told SciBX the method should be extendable to other classes of natural products.

\section{Bridging the gap}

"The paper is very impressive in its scientific complexity and in how the researchers have worked on a practical issue and resolved it with a number of biotechnological tools and techniques," said Dennis Brown, president and CSO of ChemGenex Pharmaceuticals Ltd. "This may open a new area of synthetic chemistry."

ChemGenex is focused on developing therapeutics to treat various cancers. Its lead candidate, the naturally occurring alkaloid omacetaxine, is in development for hematological malignancies.

Brown said the question of how to produce enough material in a safe, cost-effective way has curtailed the exploration and development of natural products.

"The natural products space has such beautifully diverse, complex structures and has demonstrated its potential to provide therapeutics for over a hundred years," he said. "But that space has been underexploited because of the difficulty in making those structures," many of which are considerably larger than the typical synthetic pharmaceutical.

"This paper bridges that gap by exploiting a fermentation process, and what is known about how plants make natural products, to produce the alkaloids," Brown said. "This is an important evolution, a crossover between biotech engineering and traditional synthetic approaches."

Brown also thinks the approach described in the paper could complement traditional synthetic methods. "You could create important intermediate scaffolds in larger quantities and probably more cheaply than total synthesis or natural product extraction" and then take them forward in laboratory synthesis, he said.

Additionally, Brown thought the engineered yeast platform could function "as a toolbox to create analogs of a particular scaffold and produce new libraries for drug screening-something that would be hard to do with extraction or synthetic routes."

Thus, Brown said, the platform "could serve as a foundation for product development by allowing selective investment in key pharmacophores, new chemical entities and new libraries. It could blossom into very valuable approaches to making very complicated structures."

ChemGenex's omacetaxine mepesuccinate, a plant alkaloid found in several Cephalotaxus species, is in a Phase II trial to treat Gleevecresistant chronic myeloid leukemia $(\mathrm{CML})$ in patients who have the
T3151 Bcr-Abl point mutation. The company plans to begin a rolling NDA submission for the compound in 2009. Omacetaxine has Fast Track and Orphan Drug designation in the U.S.

Novartis AG markets Gleevec imatinib, a Bcr-Abl tyrosine kinase inhibitor, for treatment of CML and gastrointestinal stromal tumors (GIST).

Brown said ChemGenex does not isolate omacetaxine directly from plants. Instead, the company uses a semisynthetic method that starts with a plant-derived precursor molecule, from which omacetaxine can be synthesized at high yields in a well-controlled process.

Tony Mills, director of business development at Phynova Group plc, agreed with Brown that the yeast platform should make the natural products space more accessible than current approaches.

"It has implications for pharmaceutical companies that have an interest in natural products and are looking for a starting material" to begin exploring that space, he said. "You could probably use it to explore the active components in a botanical mixture extracted from a plant source, then fish out those components," and carry them forward in synthetic chemistry operations.

Mills also thought that Smolke and Hawkins had clearly demonstrated the platform's utility as a research tool for characterizing the enzymes that produce pharmacologically active compounds in a particular plant.

Phynova develops botanical drugs-complex mixtures of plantderived compounds-to treat viral infections, microbial infections and cancer in accordance with the FDA's "Guidance for industry: botanical drug products." 5

The company has completed a Phase I/IIa trial of PYN17, a compound composed of four plant extracts with antioxidant, immune modulation and liver protection properties, to treat chronic HCV. The company plans to take the compound into a Phase IIb trial in the near future, though Mills declined to provide a timeline.

Phynova also has PYN6, a plant-derived antibacterial agent, in preclinical development as a topical treatment for methicillin-resistant Staphylococcus aureus (MRSA) infection and acne.

\section{Expanding in all directions}

Smolke said her group is working to improve and extend the yeast system in several ways.

One area of effort involves expanding the alkaloid space by engineering the yeast to produce alkaloid precursors upstream of norlaudanosoline. Another effort looks to expand the diversity of benzylisoquinoline alkaloids that the platform can produce, with a focus on undisclosed alkaloids.

The group is also integrating new molecular tools to improve the platform's efficiency and product yield.

Additionally, the team is exploring combinations of enzymesfrom plants and other organisms - that could produce benzylisoquinoline alkaloids that do not occur naturally. "We are interested in using our system as a research tool for functional genomics to identify and characterize new plant enzymes in this pathway, but this will be a longer-term effort," Smolke said.

Caltech has submitted patent applications for the enzyme-tuning strategy and for the production of benzylisoquinoline alkaloids in yeast. 


\section{DRUG PLATFORMS}

\section{REFERENCES}

1. Hawkins, K. \& Smolke, C. Nat. Chem. Bio.; published online Aug. 10, 2008; doi:10.1038/nchembio.105

Contact: Christina D. Smolke, California Institute of Technology,

Pasadena, Calif.

e-mail: smolke@cheme.caltech.edu

2. Minami, H. et al. Proc. Natl. Acad. Sci. USA 105, 7393-7398 (2008)

3. Hawkins, K. \& Smolke, C. J. Biol. Chem. 281, 13485-13492 (2006)

4. Zhu, W. et al. J. Immunol. 175, 7357-7362 (2005)

5. Food and Drug Administration. Guidance for industry: botanical drug products. <http://www.fda.gov/cder/guidance/4592fnl.pdf> (June 2004)

\section{COMPANIES AND INSTITUTIONS MENTIONED}

California Institute of Technology, Pasadena, Calif.

ChemGenex Pharmaceuticals Ltd. (ASX:CSX; NASDAQ:CSXP),

Geelong, Victoria, Australia

Food and Drug Administration, Rockville, Md.

Ishikawa Prefectural University, Ishikawa, Japan

Novartis AG (NYSE:NVS; SWX:NOVN), Basel, Switzerland

Phynova Group plc (LSE:PYN; Pink:PHNVY), Oxford, U.K. 